\title{
Henri-Paul Francfort, Roberte Hamayon (eds.), The Concept of Shamanism: Uses and Abuses
}

Budapest, Akadémia Kiadó, 2002, 408 p. (bibliogr.)

\section{Erwan Dianteill}

\section{OpenEdition}

\section{Journals}

Édition électronique

URL : http://journals.openedition.org/assr/825

DOI : $10.4000 /$ assr.825

ISSN : $1777-5825$

\section{Éditeur}

Éditions de l'EHESS

Édition imprimée

Date de publication : 1 octobre 2003

Pagination : 63-170

ISBN : 2-222-96739-2

ISSN : 0335-5985

\section{Référence électronique}

Erwan Dianteill, « Henri-Paul Francfort, Roberte Hamayon (eds.), The Concept of Shamanism: Uses and Abuses ", Archives de sciences sociales des religions [En ligne], 124 I octobre - décembre 2003, document 124.19, mis en ligne le 24 octobre 2005, consulté le 24 septembre 2020. URL : http:// journals.openedition.org/assr/825; DOI : https://doi.org/10.4000/assr.825 
n'assurerait-elle pas encore mieux cette fonction? Il y a dans ce chapitre une défense de la situation traditionnelle féminine dans ce mouvement hassidique. Il est vrai que les femmes Loubavitch ont leurs organisations propres très actives, que le rèbbe et autres leaders manifestent bien souvent leur estime pour elles, etc. En fait, et c'est là au sein du mouvement hassidique probablement l'originalité majeure Loubavitch en ce domaine, l'autonomie féminine y est largement soutenue, quoique toujours dans les limites d'une totale fidélité aux lois et traditions judaïques qui assurent la ségrégation déjà évoquée et une domination masculine dans les communautés.

En définitive, ce livre présente un double intérêt : d'une part il apporte des informations utiles sur Loubavitch et la politique et d'autre part, il témoigne sur la manière dont une intellectuelle venue du monde «profane "s'insère dans un mouvement hassidique, et joue le rôle malcommode d'observateur engagé.

\section{Jacques Gutwirth.}

FRANCFORT (Henri-Paul), HAMAYON (Roberte), eds.

The Concept of Shamanism: Uses and Abuses. Budapest, Akadémia Kiadó, 2002, 408 p. (bibliogr.).

Cet ouvrage collectif est le second issu de la $4^{\mathrm{e}}$ conférence de la Société Internationale de Recherches Chamaniques qui s'est tenue en 1997 à Chantilly. Le premier volume, intitulé La politique des esprits - Chamanismes et religions universalistes a été publié en 2000 par la Société d'Ethnologie à Nanterre. Le présent ouvrage est fort différent du premier dans la mesure où il est centré sur la validité du concept de chamanisme lorsqu'il est utilisé " hors contexte », c'est-à-dire hors de l'étude de certaines aires culturelles bien identifiées (les sociétés de chasseurs-cueilleurs d'Asie et d'Amérique, principalement) par la tradition anthropologique.

Après une brillante introduction générale signée par R.H., qui pose clairement les enjeux des différentes études réunies, une première partie traite de l'usage du concept de « chamanisme » en préhistoire. Elles sont en fait toutes orientées contre la thèse de Clottes et LewisWilliams (Les chamanes de la préhistoire Transe et magie dans les grottes ornées, Paris, Seuil, 1996) selon laquelle les peintures rupestres seraient un exemple d'art "chamanique " paléolithique. À peu près toutes les régions du monde sont couvertes par des spécialistes afin de démontrer l'inanité de cette thèse que H.-P.F. définit en quatre points : le cerveau humain est par nature capable d'atteindre des «états altérés de conscience »; il en dérive une religion primitive universelle associée à des visions de formes géométriques et de transformations réversibles de l'homme en animal; l'art pariétal est l'expression de cette religion; enfin, le chaman est un artiste visionnaire.

Or, les données de terrain collectées par les archéologues infirment un tel rapport entre art paléolithique et religion «primitive». Il n'existe aucune preuve empirique de ce que les hommes du paléolithique eurent de quelconques expériences de « conscience modifiée », et s'ils en ont bien eues, rien ne prouve que les peintures rupestres en sont dérivées. Le lien postulé entre ces deux activités n'est " démontré » qu'au moyen d'arguments tautologiques, qui supposent ce qu'il faut prouver. Le terme lui-même de "chaman » désigne des réalités très variables dans le temps et dans l'espace, des activités sociales que la théorie de Clottes et Lewis-Williams ne prend pas en compte. Il n'existe pas de " chamanisme universel ». Quant à l'idée que tout art a quelque chose de "chamanique», elle va contre tous les travaux en sciences sociales dans ce domaine.

Après ce salutaire exercice de critique rationnelle d'une hypothèse pour le moins incertaine, l'ouvrage réunit des contributions de nature très différente. Il s'agit cette fois d'études consacrées au « chamanisme dans les sociétés modernes ».

Le premier article de cette section, qui fait office d'introduction à ce sous-ensemble, est signé par Ulla Johansen. Il est consacré à la différence entre le chamanisme et le néochamanisme. Le chamanisme des années 1930 a-t-il survécu sans changement ? U. Johansen décrit un exemple de transformation accéléré chez les Tuvas de Sibérie. Quatre caractéristiques distinguent le chamanisme classique du néo-chamanisme qui se développe depuis la disparition de l'URSS. La première concerne le système cognitif: tandis que les chamans étaient généralement analphabètes et immergés dans une culture relativement close, les néochamans sont passés par les institutions d'enseignement soviétiques et ont un accès à des visions du monde variées, grâce à la télévision et à la presse. Ils ont aussi connaissance d'autres religions qui pénètrent dans la région : bouddhisme, orthodoxie, islam et même les doctrines protestantes et «jéhovistes». La seconde concerne la dimension internationale du néochamanisme. Alors que les chamans «classiques » exerçaient dans quelques villages 
seulement, les néo-chamans voyagent et participent même à des congrès internationaux où l'on attend d'eux le plus souvent qu'ils présentent un chamanisme conforme à l'idée qu'en a l'Occident. Le troisième point concerne les destinataires de la séance chamanique : les néochamans ne s'adressent plus nécessairement à des individus appartenant à leur propre culture. La cérémonie est plus courte, elle a lieu de jour pour permettre l'enregistrement vidéo. Enfin, le néo-chamanisme est associé à une sorte de « nationalisme» ethnique. Il s'agit apparemment de revaloriser une culture méprisée du temps de l'Union Soviétique, mais aussi d'unifier artificiellement un ensemble de pratiques qui étaient hétérogènes, même à l'échelle du pays Tuva. Les néo-chamans composent ainsi des vêtements cérémoniels à partir d'éléments variés glanés dans les musées ethnographiques. Néanmoins, U. Johansen conclut que les racines les plus profondes du chamanisme, à savoir " la croyance en l'existence des âmes et la représentation de leur apparence et de leur comportement» (p. 302), n'ont pas disparu, y compris dans le cas de certains chamans apparemment "modernes".

Parmi les textes restant du recueil, trois d'entre eux nous intéressent ici particulièrement car ils traitent, à des degrés divers, des effets de l'introduction de l'écriture sur le chamanisme.

Magali Demanget prend pour objet les transformations induites par le développement du tourisme sur le chamanisme des Mazatec du Mexique. Ici aussi, il est difficile de distinguer chamanisme "classique " et néo-chamanisme, et c'est plutôt la dynamique de transformation, la délimitation d'une frontière nette entre deux systèmes qui intéressent l'auteur. L'un des facteurs les plus importants de changement a été l'intervention dans les années 1950 d'un ethnobotaniste, Gordon Wasson, qui décrivit l'usage religieux de champignons hallucinogènes et qui rendit célèbre une chaman mazatec nommée Maria Sabina. L'ouvrage de Wasson attira beaucoup de visiteurs en quête d'expériences psychédéliques dans les années 1960 et 1970. Aujourd'hui, il s'agit d'un tourisme très assagi, mais la figure de Maria Sabina reste largement utilisée par les autorités locales et par les commerçants à des fins de promotion culturelle et économique. On propose discrètement aux touristes curieux de participer à une cérémonie chamanique: celle-ci fait dorénavant partie du complexe touristique.

Le texte de Boudewijn Walraven porte sur l'expansion de la culture écrite chez les chamans coréens. Ils ne se contentent pas de lire des ouvrages d'anthropologie, ils en écrivent aussi. Considérés pendant des siècles comme des analphabètes (ce qui était inexact), y compris par l'élite coréenne lettrée, les mudang montrent aujourd'hui qu'ils peuvent utiliser publiquement les mêmes outils que leurs détracteurs. Les publications des chamans prennent des formes assez variées, qui vont de l'autobiographie romancée à la transcription de chants rituels. Peut-on considérer ces textes comme de «nouvelles sources» pour l'étude du chamanisme coréen? s'interroge l'A.. La réponse est affirmative, si l'on ne cherche pas à identifier une quelconque "authenticité 》 chamanique, mais si l'on veut étudier ses aspects les plus contemporains. "Ces livres, écrit-il, sont intéressants pour connaître le chamanisme coréen contemporain précisément parce qu'ils témoignent des contacts avec les médias, avec le monde académique, avec les autres groupes sociaux (ce que les folkloristes 'à l'ancienne' considèrent comme un manque d'authenticité) ». Si l'anthropologie du chamanisme veut éviter de se transformer en une entreprise de conservation folklorique, elle a tout intérêt à suivre le conseil de B. Walraven pour se constituer en étude du chamanisme en modernité, sans nostalgie.

Danièle Vazeilles prend pour objet le chamanisme et le New Age chez les Sioux Lakota, qui sont eux aussi les auteurs d'ouvrages associant ces deux sources religieuses. Depuis les années 1960, le «tourisme chamanique » s'est développé chez les Sioux : des Américains anglo-saxons se rendent dans leur territoire pour participer aux cérémonies de la «Sundance», de la «sweatlodge» ou de la "Vision Quest». Les livres de Carlos Castaneda et de Michael Harner contribuèrent largement à cette recherche de spiritualité alternative, souvent liée à des thérapies de groupe. Simultanément, les médecines «douces», le végétarisme, la manipulation de cristaux, l'astrologie, l'écologie, les spiritualités orientales et la science fiction (entre autres) s'articulèrent de façon peu systématique en un discours sur l' « énergie positive » qui se trouve au cour du mouvement «New Age». Le chamanisme indien, ou plutôt l'idée que les "New Agers" s'en faisaient, a été intégré aux influences hétérogènes qui composent leurs croyances. Des ouvrages publiés par des Sioux se sont ainsi placés sur ce marché de la littérature «New Age ». Ils donnent du chamanisme une présentation acceptable pour un lecteur pénétré par les croyances "New Age», en présentant le chamanisme comme "holistique ", «écologique », "mystique ». Ces catégories n'ont pas grand sens dans le contexte social traditionnel sioux, mais par un effet en retour, on peut supposer qu'ils peuvent supplanter le 
chamanisme «classique». Danièle Vazeilles semble ainsi regretter que certains Sioux " exploitent sans vergogne leur propre culture ». Les derniers paragraphes de l'article nous font craindre que l'A. n'échappe malheureusement pas à la nostalgie folkloriste que dénonce justement $B$. Walraven.

$\mathrm{Au}$ total, aussi bien dans sa partie de préhistoire que dans celle consacrée au néochamanisme, l'ouvrage fera date. En effet, cette entreprise de critique argumentée de la notion de " chamanisme » n'est pas sans rappeler la déconstruction du concept de " totémisme » par C. Lévi-Strauss. Elle a les mêmes vertus euristiques.

\section{Erwan Dianteill.}

FRÉGOSI (Franck), WILLAIME (Jean-Paul), éds.

Le Religieux dans la commune. Les régulations locales du pluralisme religieux en France. Genève, Labor et Fides, 2001, 371 p. (tablx.) (coll. " Histoire et société »).

Cet ouvrage rassemble les travaux d'un colloque organisé en novembre 1998, à Strasbourg par le Centre de Recherche Société, Droit et Religions en Europe, sur le thème «Les régulations locales du pluralisme religieux en France ». Les contributions sont de deux natures : dix communications scientifiques, qui composent les deux parties principales de l'ouvrage et sept témoignages ou prises de positions d'acteurs politiques ou religieux, dans deux tables rondes spécifiques qui sont l'objet de contributions plus brèves.

F.F. introduit le propos en montrant que les régulations locales du pluralisme religieux correspondent à trois processus : les modes de perception et d'appréhension du pluralisme religieux local, les modes d'insertion des groupes religieux dans la réalité religieuse locale et les modes et types de prise en compte effective du pluralisme religieux dans et par les politiques municipales.

La première partie porte sur «les collectivités locales face au pluralisme religieux ». La première contribution fait le point sur les relations juridiques entre les groupements religieux et les collectivités locales, en droit général et en droit alsacien-mosellan (Francis Messner). L'auteur montre que si la législation des cultes offre des ressources non négligeables, la «nonexistence d'un statut des cultes », remplacé à partir de 1905 par un "ensemble de mécanismes isolés susceptibles d'être appliqués aux religions qui en font la demande ", oblige les groupes religieux à solliciter une procédure de " reconnaissance » pour chaque type de mécanisme de soutien. Les problèmes inédits liés à la gestion du patrimoine des anciens groupes religieux et aux demandes de soutien des nouveaux groupes religieux laissent souvent les maires dans l'embarras.

Les effets concrets de la pluralisation religieuse à l'échelle locale sont abordés dans la présentation d'une enquête menée auprès des maires de 334 communes du Bas-Rhin (Solange Wydmush). L'A. montre que le pluralisme est plus facilement pratiqué en territoire protestant que catholique et que les relations des communes avec les dissidences chrétiennes sont plus difficiles qu'avec les religions non chrétiennes (islam et bouddhisme). En outre, en situation de concurrence, les groupes religieux, reconnus ou non, soignent davantage leurs relations avec la municipalité. Réciproquement, un cinquième des maires ayant des cultes non reconnus présents sur leur commune affirment avoir pris des initiatives inter-religieuses.

La gestion municipale de l'islam dans de grandes villes est abordée par l'étude comparée de Mulhouse et de Strasbourg (F.F.). Ces deux cas révèlent à la fois le volontarisme d'équipes municipales face aux mauvaises conditions de l'exercice du culte musulman, et l'inadéquation de la volonté de "subordonner l'amélioration des conditions de pratiques du culte à l'émergence d'un interlocuteur unique ». L'expérience de Mulhouse montre aussi l'échec d'une volonté de recherche de l'islam et du musulman «idéologiquement correct» et de la tentation pour la mairie d'organiser un islam républicain. Dans les deux villes, les municipalités se sont finalement tournées vers une gestion plus pragmatique de l'islam en renonçant au modèle centralisé et en facilitant progressivement des implantations de proximité.

Le cas d'un groupe religieux socialement controversé est abordé à travers l'implantation du siège européen de la Sokka Gakaï, dans une petite commune des Bouches-du-Rhône (Raphaël Liogier). L'A. montre comment après une période de bons rapports avec la population et la municipalité, la situation change totalement suite à la publication du rapport Vivien (1985), qui classe le groupe dans la catégorie « sectes dangereuses".

Enfin, le cas de Marseille-Espérance est présenté comme une gestion originale des relations entre la mairie et les responsables religieux, par le biais d'une structure présidée par le maire, mais n'ayant aucun cadre juridique, et qui mène cependant à une forme de régulation symbolique par le biais de déclarations, de 